EUROPEAN ORGANIZATION FOR NUCLEAR RESEARCH

European Laboratory for Particle Physics

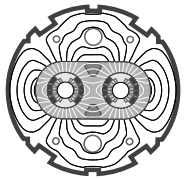

Large Hadron Collider Project

LHC Project Report 115

\title{
Surface Resistance Measurements for the LHC Beam Screen
}

Caspers, F.; Morvillo, M.; Ruggiero, F.

\begin{abstract}
A critical review of resistive losses in the LHC beam screen, taking into account anomalous skin effect and surface roughness, has triggered a programme of surface resistance measurements at different temperatures, frequencies and magnetic field intensities. The aim is to establish a realistic heating budget for the LHC cryogenic system and to optimize the fabrication process for the copper coating of the beam screen. Preliminary results at cryogenic temperatures (without magnetic field) indicate a surface resistance about a factor two larger than previously estimated: an absolute measurement precision of a few per cent is reached by comparing the quality factors of even and odd TEM modes in a cylindrical structure with two inner conductors.
\end{abstract}

SL-AP
PS
Presented at 1997 Particle Accelerator Conference, Vancouver, 12-16 May 1997.

Administrative Secretariat

LHC Division

CERN

$\mathrm{CH}$ - 1211 Geneva 23

Switzerland

Geneva, 12 juin 1997 


\title{
Surface Resistance Measurements for the LHC Beam Screen
}

\author{
F. Caspers, M. Morvillo and F. Ruggiero, European Laboratory for Particle Physics (CERN)
}

\section{Abstract}

A critical review of resistive losses in the LHC beam screen, taking into account anomalous skin effect and surface roughness, has triggered a programme of surface resistance measurements at different temperatures, frequencies and magnetic field intensities. The aim is to establish a realistic heating budget for the LHC cryogenic system and to optimize the fabrication process for the copper coating of the beam screen. Preliminary results at cryogenic temperatures (without magnetic field) indicate a surface resistance about a factor two larger than previously estimated: an absolute measurement precision of a few per cent is reached by comparing the quality factors of even and odd TEM modes in a cylindrical structure with two inner conductors.

\section{INTRODUCTION}

The LHC beam screen is a stainless steel pipe (for mechanical strength during quench) with a $50 \mu \mathrm{m}$ copper layer coated on its inner surface. It is cooled at a temperature ranging from 5 to $20 \mathrm{~K}$. The purpose of the copper coating is to reduce the surface resistance $R_{s}$, i.e., the ratio between longitudinal component of the electric field at the wall surface and beam-induced wall current. This is important to minimize at the same time transverse resistive wall instability (a relatively low frequency phenomenon) and beaminduced wall heating. At top energy and for nominal beam parameters, the classically computed resistive wall heating of the LHC beam screen is $75 \mathrm{~mW} / \mathrm{m}$. Owing to the anomalous skin effect, this value should increase by $11 \%$, but according to surface resistance measurements performed on samples of the SSC beam tube, the increase could be significantly larger [1].

For a circular screen of radius $b$, the parasitic heating power per unit length is given by

$$
\frac{P}{L}=\frac{I_{\mathrm{av}}^{2}}{M f_{0}} \cdot \frac{c^{2}}{\pi} \int_{0}^{\infty}|\tilde{\lambda}(\omega)|^{2} \frac{R_{s}(\omega)}{2 \pi b} d \omega,
$$

where $I_{\text {av }}$ is the average beam current, $M$ the number of bunches, $f_{0}$ the revolution frequency, $c$ the velocity of light and $\tilde{\lambda}(\omega)$ the bunch spectrum, which for the relatively short LHC bunches at $7 \mathrm{TeV}(7.5 \mathrm{~cm}$, r.m.s. $)$ will cover a wide high frequency region (637 MHz, r.m.s.).

Since the parasitic heating scales with the square of $I_{\text {av }}$, for the ultimate beam intensity of $850 \mathrm{~mA}$, it may become comparable to the synchrotron radiation power loss of $326 \mathrm{~mW} / \mathrm{m}$. Reliable data for the surface resistance up to a few $\mathrm{GHz}$ is therefore needed to establish a realistic heating budget for the cryogenic system and to optimize the fabrication process for the copper coating of the beam screen. The situation is complicated by the fact that the anomalous skin effect is accompanied by a strong magnetic field and by phenomena associated with the surface roughness. There is no valid theory for estimating the surface resistance in this complex environment and this has triggered a programme of surface resistance measurements on several samples of the LHC beam screen.

\section{EXPERIMENTAL SETUP}

To measure the surface resistance at several temperatures and with an $8.4 \mathrm{~T}$ magnetic field, we decided to avoid using supercondutors. With the experimental setup shown in Fig. 1, we measure the quality factors $Q^{++}$and $Q^{+-}$of even and odd modes excited in a $1 \mathrm{~m}$ long cylindrical TEM mode cavity with two inner conductors. Relying on the only assumption that $R_{s}$ be the same for the two inner conductors, we then obtain the surface resistance of both inner conductors and outer tube in steps of about $150 \mathrm{MHz}$.

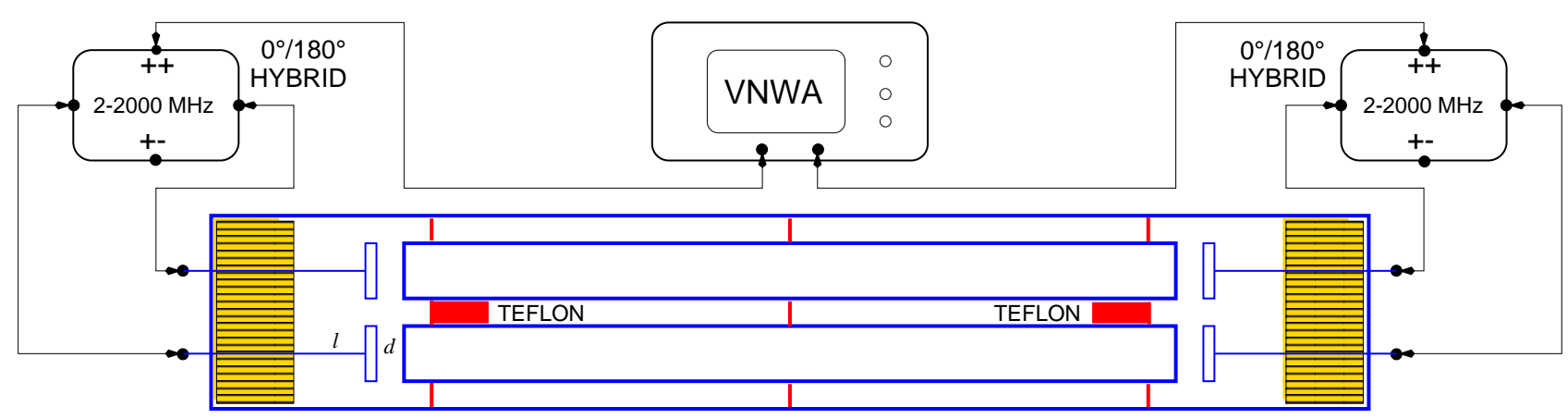

Figure 1: Experimental setup: a vector network analyser (VNWA) is used in conjunction with two hybrids, yielding either even (++) or odd (+-) mode excitation for a $1 \mathrm{~m}$ long cylindrical cavity with two inner conductors, held by 3 teflon supports. The near degeneracy of even and odd modes is removed by two teflon splitters placed between the inner conductors. 
a) $R_{s}[m \Omega] \mathrm{IN}\left(\mathrm{RRR} \simeq 200, \rho(300 \mathrm{~K})=2 \times 10^{-8} \Omega \mathrm{m}\right)$

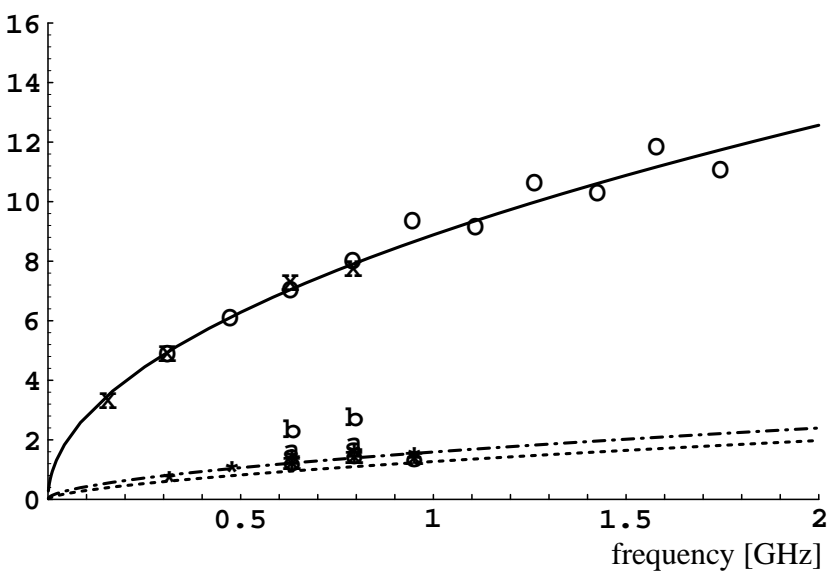

c) $R_{s}[m \Omega] \mathrm{IN}\left(\mathrm{RRR} \simeq 200, \rho(4 \mathrm{~K})=10^{-10} \Omega \mathrm{m}\right)$

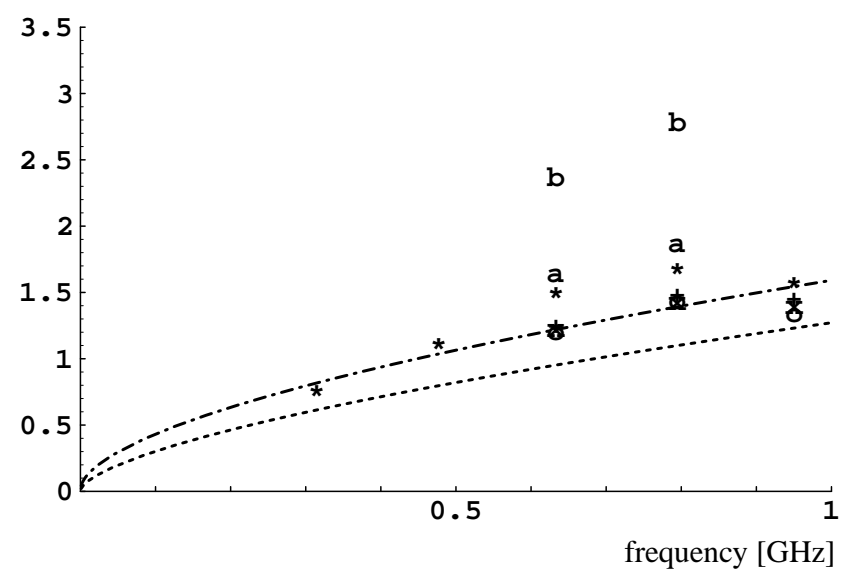

e) $R_{s}[m \Omega] \mathrm{IN}\left(\mathrm{RRR} \simeq 200, \rho(4 \mathrm{~K})=10^{-10} \Omega \mathrm{m}\right)$

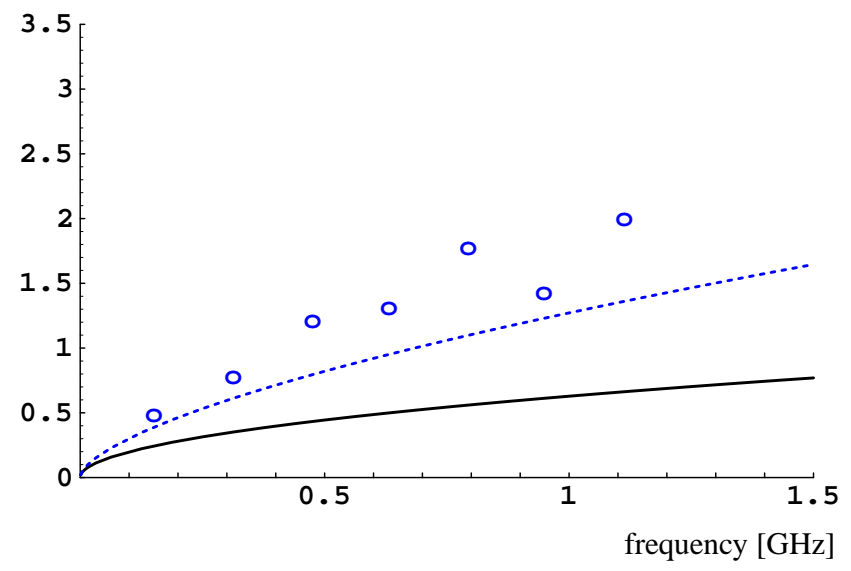

b) $R_{s}[m \Omega]$ OUT $\left(\mathrm{RRR} \simeq 50, \rho(300 \mathrm{~K})=3.2 \times 10^{-8} \Omega \mathrm{m}\right)$

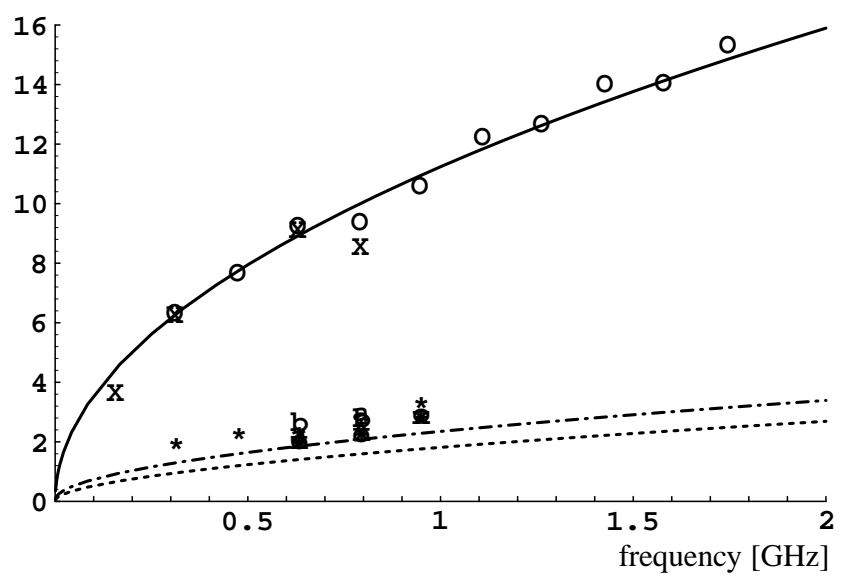

d) $R_{s}[m \Omega]$ OUT $\left(\mathrm{RRR} \simeq 50, \rho(4 \mathrm{~K})=6.4 \times 10^{-10} \Omega \mathrm{m}\right)$

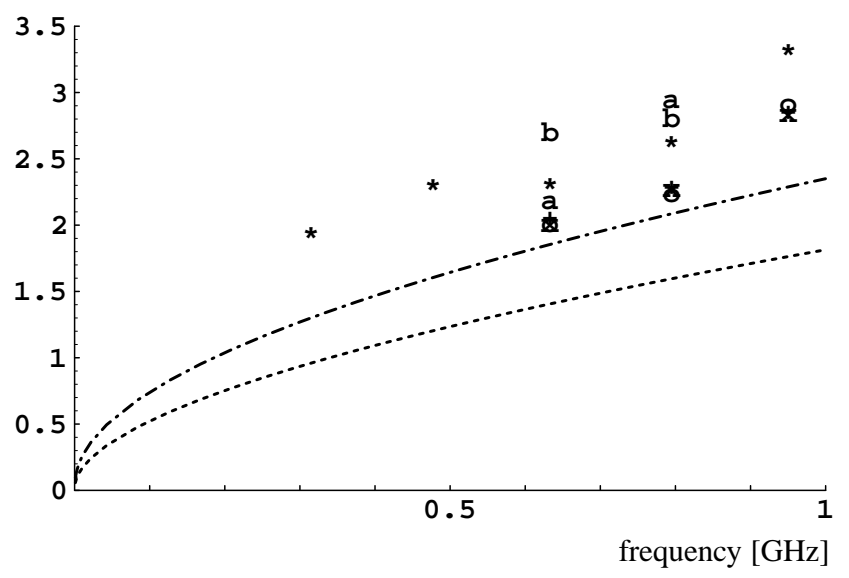

f) $R_{s}[m \Omega]$ OUT $\left(\mathrm{RRR} \simeq 50, \rho(4 \mathrm{~K})=6.4 \times 10^{-10} \Omega \mathrm{m}\right)$

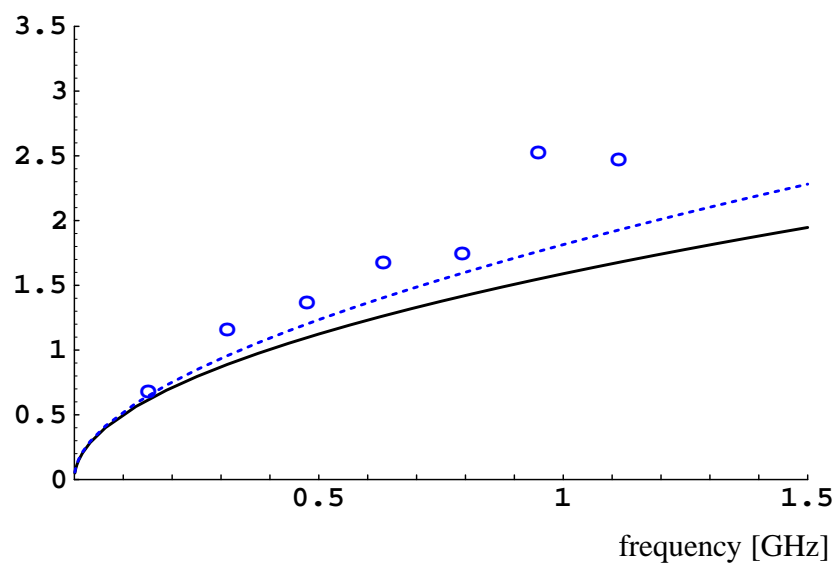

Figure 2: Surface resistance measured for the thermally treated inner conductors (a, c, e) having a dc RRR $\simeq 200$, and for the outer tube $(\mathrm{b}, \mathrm{d}, \mathrm{f}$ ) with no thermal treatment and a dc RRR 250 , compared to the predictions in the classic (solid line) and anomalous regime (dot-dashed lines: $40 \mathrm{~K}$, dashed lines: $4 \mathrm{~K}$ ). Figures (a) and (b) show data measured at room temperature, either before ("X") or after cryogenic measurements using shorter coupling networks ("O"), at $40 \mathrm{~K}$, either before ("b") or after ("a") injection of He gas during cool down (leading to a sudden thermalisation of the inner conductors, otherwise thermally insulated) or with external heating (“*”), at $20 \mathrm{~K}$ (“+”), at $10 \mathrm{~K}$ ("x") and at 4 K ("o"). Some measurements at $40 \mathrm{~K}$ have a large temperature spread from top to bottom of the outer tube. The data at room temperature is best fitted assuming a resistivity $\rho(300 \mathrm{~K})=2 \times 10^{-8} \Omega \mathrm{m}$ for the inner conductors and $\rho(300 \mathrm{~K})=3.2 \times 10^{-8} \Omega \mathrm{m}$ for the outer tube. Figures (c) and (d) are a magnification of (a) and (b), respectively, showing only measurements at cryogenic temperatures. The data in (e) and ( $\mathrm{f}$ ) has been obtained at $4 \mathrm{~K}$ with shorter coupling networks, to allow measurements in a wider frequency range: data above $1.2 \mathrm{GHz}$, however, has been discarded due to strong or badly corrected coupling. 
a) $R_{s}[m \Omega] \mathrm{IN}\left(\mathrm{RRR} \simeq 200, \rho(4 \mathrm{~K})=1.35 \times 10^{-10} \Omega \mathrm{m}\right)$

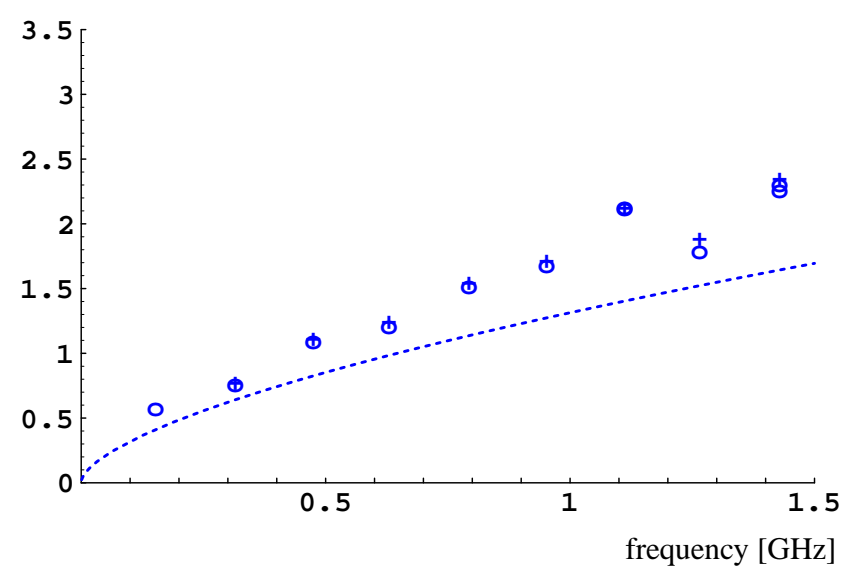

b) $R_{s}[m \Omega]$ OUT $\left(\mathrm{RRR} \simeq 200, \rho(4 \mathrm{~K})=1.6 \times 10^{-10} \Omega \mathrm{m}\right)$

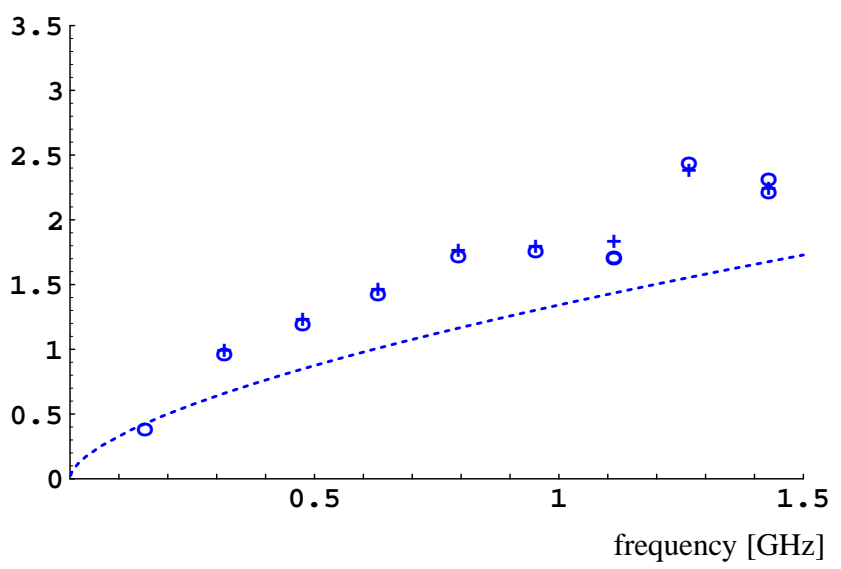

Figure 3: Surface resistance measured with shorter coupling networks at $4 \mathrm{~K}$ ("o") and at $20 \mathrm{~K}$ ("+") for the inner conductors (a) and the outer tube (b), both thermally treated at $350^{\circ} \mathrm{C}$ and having a measured dc RRR $\simeq 200$, compared to the predictions in the anomalous regime (dashed lines). The corresponding data at room temperature (not shown in the figure) is best fitted assuming a resistivity $\rho(300 \mathrm{~K})=2.7 \times 10^{-8} \Omega \mathrm{m}$ for the inner conductors and $\rho(300 \mathrm{~K})=$ $3.2 \times 10^{-8} \Omega \mathrm{m}$ for the outer tube. The slightly higher resistivity attributed to the inner conductors may be due to small scratches in their copper coating, caused by the sliding teflon supports repeatedly mounted during previous measurements.

Indeed the unloaded quality factor of each mode, given by $1 / Q=\Gamma_{\mathrm{IN}} / R_{s}^{\mathrm{IN}}+\Gamma_{\mathrm{OUT}} / R_{s}^{\mathrm{OUT}}$, is related through calculable geometric coefficients [2] $\Gamma_{\text {IN }}$ and $\Gamma_{\text {OUT }}$ to the surface resistances $R_{s}^{\mathrm{IN}}$ and $R_{s}^{\mathrm{OUT}}$ of the inner conductors and of the outer tube. Measuring $Q^{++}$and $Q^{+-}$, therefore, we can solve a system of two equations in the two unknowns $R_{s}^{\mathrm{IN}}$ and $R_{s}^{\mathrm{OUT}}$.

The two inner cylindrical conductors (of radius $8 \mathrm{~mm}$ ) and the outer tube (of radius $19.6 \mathrm{~mm}$ ) are all made out of stainless steel with a $50 \mu \mathrm{m}$ electrodeposited copper layer, having a surface roughness of a few $\mu \mathrm{m}$. Future measurements are planned also with a colaminated copper layer. The distance between the axes of the two inner conductors is $19.5 \mathrm{~mm}$. The distance $d$ between each coupling network and the inner conductors (a few $\mathrm{cm}$ ) is adjusted to have sufficiently weak coupling coefficients $\beta_{1}(\omega)$ and $\beta_{2}(\omega)$ over the frequency range of interest. We calculate coupling by measuring reflection coefficients at each coupling port, while the loaded quality factor $Q_{\text {load }}(\omega)$ for each mode is obtained by a transmission coefficient measurement. The corresponding unloaded quality factor is then given by $Q(\omega)=\left[1+\beta_{1}(\omega)+\beta_{2}(\omega)\right] Q_{\text {load }}(\omega)$. The length $l$ of the coupling networks has been varied from 12 down to $4 \mathrm{~cm}$, to allow measurements in a wider frequency range (up to about $1.5 \mathrm{GHz}$ ). Indeed coupling grows with frequency, but suddenly drops over a large frequency gap whose location depends critically on $l$. This phenomenon seems related to internal resonances of the coupling networks.

\section{PRELIMINARY EXPERIMENTAL RESULTS}

In Figs. 2 and 3 we present some preliminary surface resistance measurements without magnetic field, from room temperature down to $4.2 \mathrm{~K}$, compared to the predictions in the classic and anomalous regime. The inner conductors have been thermally treated at $350^{\circ} \mathrm{C}$ and have a measured dc RRR around 200. Fig. 2 refers to an outer tube with no thermal treatment and a dc RRR $\simeq 50$, while Fig. 3 refers to a thermally treated outer tube. The low temperature resistivity of the outer tube in the case of Fig. 2 is comparable to that of the LHC beam screen in a magnetic field of $8.4 \mathrm{~T}$, but the measured surface resistance around $1 \mathrm{GHz}$ is almost a factor two larger than previously estimated.

Both figures show a slight modulation of the surface resistance as a function of frequency; this modulation is anticorrelated for the IN versus OUT data and grows from a few per cent at low frequencies to about $10 \%$ beyond $1 \mathrm{GHz}$. We are currently investigating this effect, which might be due to the teflon supports, to the teflon splitters, to geometric imperfections or to the coupling networks.

\section{ACKNOWLEDGEMENTS}

We would like to thank several CERN colleagues who gave significant contributions to these measurements and, in particular, A. Poncet and C. Benvenuti for useful discussions of the experimental setup, A. Mathewson and N. Kos (beam screen prototypes), S. Sgobba (surface metrology and welding), C. Reymermier and L. Nikitina (designs), J. Rieubland, G. Ferlin and A. Jacquemot for their constant help in the cryolab, L. Walckiers and A. Arn (measurements in progress with magnetic field).

\section{REFERENCES}

[1] W. Chou and F. Ruggiero, LHC Project Note 2 (1995).

[2] G.S. Smith and J.D. Nordgård, IEEE Trans. Microwave Theory Tech., vol. MTT-28, pp. 887-893, Aug. 1980. 